\title{
Detección de genes que codifican hemolisinas en cepas de Staphylococcus aureus aisladas en pantallas de teléfonos móviles de estudiantes de último año de odontología en Cuenca-Ecuador, 2020-2021.
}

\author{
Detection of hemolysin-encoding genes in Staphylococcus aureus strains isolated \\ from cell phone screens of senior dental students in Cuenca-Ecuador, 2020-2021.
}

\author{
Nasly Hurtado Cantos,* Paola Orellana B, ${ }^{\ddagger}$ Carlos Andrade Tacuri ${ }^{\S}$
}

\section{RESUMEN}

Staphylococcus aureus es la especie más común implicada en las enfermedades infecciosas que causan morbilidad y mortalidad a nivel mundial. Posee los genes $h l a, h l b, h l d, h l g, h l g-v$ que codifican para hemolisinas. Las hemolisinas son reconocidas como un factor de virulencia potencial que ataca a la membrana y produce destrucción de las plaquetas y necrosis. Tienen la capacidad de sobrevivir por largos periodos en superficies inertes como en pantallas de teléfonos móviles. Estudio observacional de tipo transversal descriptivo. Se aislaron en un estudio previo 16 cepas de Staphylococcus aureus a partir de 92 muestras de pantallas de teléfonos móviles de estudiantes de odontología. Se utilizó la técnica de reacción en cadena de la polimerasa para detectar los genes que codifican para hemolisinas. El gen hla se detectó en $75 \%$ (12/16) de cepas de Staphylococcus aureus, hlb en 25\% (4/16), hld 75\% (12/16), hlg 75\% (12/16), hlg-v 13\% (2/16). Este estudio evidencia el alto porcentaje de cepas virulentas que poseen genes que codifican para hemolisinas en pantallas de teléfonos móviles, lo que puede contribuir a la diseminación de este patógeno. Es imperioso implementar medidas para la desinfección de teléfonos móviles.

Palabras clave: Staphylococcus aureus, hemolisinas, teléfonos móviles.

\section{ABSTRACT}

Staphylococcus aureus is the most common species implicated in infectious diseases causing morbidity and mortality worldwide. It has the hla, hlb, hld, hlg, hlg-v genes encoding for hemolysins. Hemolysins are recognized as a potential virulence factor that attacks the membrane and causes platelet destruction and necrosis. They have the ability to survive for long periods on inert surfaces such as cell phone screens. Observational descriptive cross-sectional study. Sixteen strains of Staphylococcus aureus were isolated in a previous study from 92 samples of cell phone screens of dental students; the polymerase chain reaction technique was used to detect genes coding for hemolysins. The hla gene was detected in 75\% (12/16) of Staphylococcus aureus strains, hlb in 25\% (4/16), hld 75\% (12/16), hlg 75\% (12/16), hlg-v 13 $\%(2 / 16)$. This study evidences the high percentage of virulent strains that possess genes encoding for hemolysins in cell phone screens, which may contribute to the dissemination of this pathogen. It is imperative to implement measures for the disinfection of cell phones.

Keywords: Staphylococcus aureus, hemolysins, cell phones.

\footnotetext{
* Egresada de la Unidad Académica de Salud y Bienestar, Carrera de Odontología, Universidad Católica de Cuenca. Cuenca-Ecuador. ORCID: 0000-0001-8285-3258.

‡ Unidad Académica de Salud y Bienestar, Carrera de Odontología, Laboratorio de Genética y Biología Molecular del Centro de Investigación, Innovación y Transferencia de Tecnología. Universidad Católica de Cuenca. Cuenca-Ecuador. ORCID: 0000-0001-6276-0521.

§ Unidad Académica de Salud y Bienestar, Carrera de Odontología, Laboratorio de Genética y Biología Molecular del Centro de Investigación, Innovación y Transferencia de Tecnología. Universidad Católica de Cuenca. Cuenca-Ecuador. ORCID: 0000-0003-3983-1314.
}

Recibido: 25 de junio de 2019. Aceptado: 09 de noviembre de 2021.

Citar como: Hurtado CN, Orellana BP, Andrade TC. Detección de genes que codifican hemolisinas en cepas de Staphylococcus aureus aisladas en pantallas de teléfonos móviles de estudiantes de último año de odontología en Cuenca-Ecuador, 2020-2021. Rev ADM. 2021; 78 (6): 332-338. https:// dx.doi.org/10.35366/102974 


\section{INTRODUCCIÓN}

L os equipos electrónicos tienen superficies de plástico y de vidrio, irregulares o lisas que sirven como fómites en la transmisión de microorganismos patógenos dentro de los hospitales, estos dispositivos están en contacto con las manos del personal de salud y no se realiza la desinfección de los mismos. ${ }^{1}$

En los teléfonos móviles se han identificado algunos tipos de bacterias y hongos. Estas bacterias pueden adherirse a estos dispositivos por medio de moléculas en sus membranas; posteriormente a su adhesión, forman un biofilm, metabolizan los componentes del plástico y los usan como nutrientes. El teléfono móvil se ha vuelto cotidiano en el ámbito social y profesional, se utiliza sin restricciones ni cuidados para desinfectarlo, por lo tanto, se transforma en un reservorio de bacterias y posibilita la transmisión de microorganismos para el desarrollo de enfermedades. ${ }^{1,2}$

Las infecciones pueden ser: nosocomiales, aquéllas que aparecen por el ingreso a hospitales, relacionadas con los cuidados sanitarios, o adquiridas en la comunidad. Este tipo de infecciones es uno de los problemas importantes en salud pública con gran importancia económica y social, por lo tanto, es necesario conocer el impacto de estas infecciones en cada paciente. ${ }^{3}$

El género Staphylococcus son bacterias de tipo cocos, anaerobios facultativos, Gram positivos, no esporulados, halófilos, inmóviles y catalasa-positivos. Estas bacterias tienen una gran resistencia a la sequedad y a la desinfección, poseen un metabolismo fermentativo y oxidasa-negativo. Son parte de la microbiota de la piel, tracto urogenital, tracto respiratorio y transitoriamente del tracto digestivo tanto en los humanos como en animales. ${ }^{4}$

Staphylococcus aureus (S. aureus) es la especie más común implicada en las enfermedades infecciosas que causan morbilidad y mortalidad a nivel mundial. ${ }^{5}$

Esta bacteria es responsable de muchas infecciones de la piel, sepsis y neumonía, tejidos blandos, endocarditis y osteomielitis. ${ }^{6}$

S. aureus puede causar patologías de diversas gravedades, éstas dependen de factores de virulencia y de su resistencia a los antibióticos.

S. aureus produce proteínas bacterianas con actividad súper antigénica: toxinas exfoliativas, toxinas del síndrome del shock tóxico, enterotoxinas, hemolisinas, arginina desaminasa y leucocidina de Panton-Valentine (PVL) que actúan como factores de virulencia. Las hemolisinas degradan los tejidos del huésped para convertirlos en nutrientes para las bacterias, son reconocidas como un factor de virulencia potencial que ataca a la membrana celular y produce la destrucción de plaquetas, destrucción lisosomal, isquemia y necrosis, ya que son importantes en el desarrollo de fiebre, impétigo, septicemia, y más patologías graves. ${ }^{7,8}$

Investigar la presencia de $S$. aureus portador de hemolisinas en teléfonos móviles puede ayudar a establecer el papel que desempeña esta clase de equipo como fuente de transmisión y reservorio de cepas virulentas de este tipo de patógeno en las clínicas odontológicas. Por lo antes mencionado, este estudio se plantea como objetivo identificar los genes que codifican para hemolisinas en cepas de Staphylococcus aureus en pantallas de teléfonos móviles de los estudiantes de último año de odontología mediante la reacción de cadena polimerasa (PCR) en Cuenca, Ecuador, 2020-2021. Los objetivos específicos fueron identificar los genes que codifican para hemolisinas hla, hlb, hld, hlg y hlg-v.

\section{MATERIAL Y MÉTODOS}

Diseño y tipo: esta investigación fue de tipo descriptivo no experimental. El diseño es de tipo observacional y corte transversal.

Muestra: los teléfonos móviles seleccionados para el estudio fueron los que pertenecían a los alumnos del último año de la carrera de odontología que asisten a la Clínica Universitaria Odontológica de Cuenca, Ecuador. Se recolectó un total de 92 muestras mediante hisopados de pantallas de teléfonos móviles sin estrategia de muestreo, ya que nuestro estudio es a conveniencia, de las cuales 16 muestras fueron positivas para Staphylococcus aureus (estudio previo). En estas muestras se analizaron los genes que codifican para hemolisinas en el periodo de septiembre de 2020 a enero de 2021.

Las variables consideradas en esta investigación son de tipo cualitativo y fueron: el nivel estudio de los estudiantes de odontología y los genes de $S$. aureus que codifican para hemolisinas (hla, hlb, hld, hlg, hlg-v) aisladas en las pantallas de los teléfonos móviles.

Durante la muestra se indicó a los estudiantes que no limpiaran la pantalla de su teléfono móvil para evitar falsos negativos.

Para la inclusión de este estudio los teléfonos móviles debían pertenecer a los alumnos de último año de la carrera de odontología que asisten a la clínica odontológica, y firmar el consentimiento informado aceptando participar en este estudio. Se excluyen de este estudio aquellos alumnos que no aceptan participar en el mismo y por lo tanto, firman el consentimiento informado. 
Las muestras fueron recolectadas en un tubo que contenía caldo soya tripticasa (CST) mediante un hisopo estéril (embebido en CST), el cual se frotó sobre la pantalla del teléfono móvil. Las muestras se llevaron al Laboratorio de Biología Molecular y Genética, Basílica, CITT de la Universidad Católica de Cuenca para su procesamiento.

Metodología: se aislaron en un estudio previo 16 cepas de Staphylococcus aureus mediante pruebas fenotípicas y genotípicas a partir de 92 muestras de pantallas de teléfonos móviles, las cuales se encontraban almacenadas a $-80{ }^{\circ} \mathrm{C}$. Estas cepas fueron incubadas por 24 horas a $37^{\circ} \mathrm{C}$ en aerobiosis, luego se sembraron en agar manitol salado y se incubaron durante $24-48$ horas a $35-37{ }^{\circ} \mathrm{C}$ en aerobiosis. ${ }^{9}$

Extracción de DNA bacteriano: con las colonias puras de $S$. aureus en agar manitol se realizó la extracción de DNA mediante el método denominado «lisis alcalina», en el cual se usa la centrifugación, alta temperatura (100 ${ }^{\circ} \mathrm{C}$ ) y una solución de lisis (sodio dodecil sulfato $1 \%$ en $\mathrm{NaOH} 0,25 \mathrm{~N}$ ) para extraer el DNA bacteriano.

Detección genotípica para hemolisinas (hla, hlb, $\mathbf{h l d}, \mathbf{h l g}, \mathbf{h l g}-\mathbf{v})$ : para la detección molecular de los genes $h l a, h l b, h l d, h l g$, hlg-v que codifican para hemolisinas se utilizó la técnica de PCR, que se detalla en la Tabla 1, se

Tabla 1: Genes, primers, cepas y condición de reacción en cadena de la polimerasa (PCR).

\begin{tabular}{|c|c|c|c|c|c|}
\hline Toxinas & Genes & Primers (5’-3') & $\begin{array}{l}\text { Tamaño del } \\
\text { amplicón }\end{array}$ & $\begin{array}{l}\text { Cepas } \\
\text { ATCC }\end{array}$ & $\begin{array}{l}\text { Condición de } \\
\text { PCR }\end{array}$ \\
\hline $\begin{array}{l}\text { Alfa- } \\
\text { hemolisina }\end{array}$ & hla & $\begin{array}{l}\text { F: CTGATTACTATCCAAGAAATTCGATTG } \\
\text { R: CTTTCCAGCCTACTTTTTTATCAGT }\end{array}$ & 209 pb & 25923 & $\begin{array}{c}5 \min 94^{\circ} \mathrm{C} \\
30 \text { ciclos: } \\
30 \text { s } 94^{\circ} \mathrm{C} \\
1 \min 55^{\circ} \mathrm{C} \\
1 \min 72^{\circ} \mathrm{C} \\
10 \min 72^{\circ} \mathrm{C}\end{array}$ \\
\hline $\begin{array}{l}\text { Beta- } \\
\text { hemolisina }\end{array}$ & $h l b$ & $\begin{array}{l}\text { F: GTGCACTTACTGACAATAGTGC } \\
\text { R: GTTGATGAGTAGCTACCTTCAGT }\end{array}$ & 309 pb & 25923 & $\begin{array}{c}5 \min 94^{\circ} \mathrm{C} \\
30 \text { ciclos: } \\
30 \text { s } 94^{\circ} \mathrm{C} \\
1 \min 55^{\circ} \mathrm{C} \\
1 \min 72^{\circ} \mathrm{C} \\
10 \min 72^{\circ} \mathrm{C}\end{array}$ \\
\hline $\begin{array}{l}\text { Delta- } \\
\text { hemolisina }\end{array}$ & hld & $\begin{array}{l}\text { F: AAGAATTTTTATCTTAATTAAGGAAGGAGTG } \\
\text { R: TTAGTGAATTTGTTCACTGTGTCGA }\end{array}$ & $111 \mathrm{pb}$ & 25923 & $\begin{array}{c}5 \min 94^{\circ} \mathrm{C} \\
30 \text { ciclos: } \\
30 \text { s } 94{ }^{\circ} \mathrm{C} \\
1 \min 55^{\circ} \mathrm{C} \\
1 \min 72^{\circ} \mathrm{C} \\
10 \min 72^{\circ} \mathrm{C}\end{array}$ \\
\hline $\begin{array}{l}\text { Gamma- } \\
\text { hemolisina } \\
\text { componentes } \\
\text { A, B y C }\end{array}$ & hlg & $\begin{array}{l}\text { F: GTCAYAGAGTCCATAATGCATTTAA } \\
\text { R: CACCAAATGTATAGCCTAAAGTG }\end{array}$ & $535 \mathrm{pb}$ & 33592 & $\begin{array}{c}5 \min 94^{\circ} \mathrm{C} \\
30 \text { ciclos: } \\
30 \text { s } 94^{\circ} \mathrm{C} \\
1 \min 55^{\circ} \mathrm{C} \\
1 \min 72^{\circ} \mathrm{C} \\
10 \min 72^{\circ} \mathrm{C}\end{array}$ \\
\hline $\begin{array}{l}\text { Gamma- } \\
\text { hemolisina } \\
\text { (variante) }\end{array}$ & $\begin{array}{l}h l g-v \\
(h l g-2)\end{array}$ & $\begin{array}{l}\text { F: GACATAGAGTCCATAATGCATTYGT } \\
\text { R: ATAGTCATTAGGATTAGGTTTCACAAAG }\end{array}$ & $390 \mathrm{pb}$ & 25923 & $\begin{array}{c}5 \min 94^{\circ} \mathrm{C} \\
30 \text { ciclos: } \\
30 \text { s } 94{ }^{\circ} \mathrm{C} \\
1 \min 55^{\circ} \mathrm{C} \\
1 \min 72^{\circ} \mathrm{C} \\
10 \min 72^{\circ} \mathrm{C}\end{array}$ \\
\hline
\end{tabular}


Tabla 2: Frecuencia de los genes que codifican para hemolisinas.

\begin{tabular}{lcr} 
Genes & Positivo, n (\%) & Negativo, n (\%) \\
\hline hla & $11(69)$ & $5(31)$ \\
$h l b$ & $4(25)$ & $12(75)$ \\
$h l d$ & $12(75)$ & $4(25)$ \\
$h l g$ & $12(75)$ & $4(25)$ \\
$h l g-v$ & $2(13)$ & $14(87)$ \\
\hline
\end{tabular}

indican los iniciadores (primers), productos amplificados, cepas ATCC ${ }^{\circledR}$ y las condiciones de amplificación. ${ }^{10}$

Todas las reacciones de PCR para todos los genes antes mencionados se llevaron a cabo en un volumen final de $20 \mu \mathrm{L}$, el cual contenía: $10 \mu \mathrm{L}$ de Mastermix Promega Green GoTaq $2 \mathrm{X}, 1.5 \mu \mathrm{L}$ de DNA y $5.5 \mu \mathrm{L}$ de agua ultrapura, $1.5 \mu \mathrm{L}$ de cada primer.

La amplificación se llevó a cabo en un termociclador Agilent Sure Cycler 8800, según los protocolos indicados mediante la Tabla 1. Los amplificados de PCR se separaron por electroforesis en geles de agarosa a $2 \% \mathrm{p} / \mathrm{v}$ (50 $\mathrm{mL}$ de gel con $2 \mu \mathrm{L}$ de SYBR Safe DNA Gel Stain 10,000x de Invitrogen) sobre una cámara horizontal inmerso en buffer TAE $1 \mathrm{X}$ con un protocolo de $70 \mathrm{~V}, 70^{\mathrm{a}}$ y $50 \mathrm{~W}$ por 60 minutos. Se usó un marcador de peso molecular de $1 \mathrm{~kb}$ Plus DNA Ladder (Trackit de Invitrogen), el tamaño de los amplificados se calculó según las migraciones en los geles de agarosa comparándolo con la migración de las bandas marcadoras del DNA del Ladder, se observaron sobre un transiluminador UV y se fotografiaron.

Análisis estadístico: los resultados de esta investigación se presentan en frecuencias absolutas y relativas. En este estudio no se formaron subgrupos de las muestras, ya que se pretendía conocer de manera global la presencia de hemolisinas (hla, hlb, hld, hlg, hlg-v) en S. aureus en las pantallas de teléfonos móviles de los estudiantes de odontología.

\section{RESULTADOS}

En este estudio se identificaron los genes $(h l a, h l b, h l d, h l g$, h/g-v) que codifican para hemolisinas de las 16 muestras de $S$. aureus que fueron aisladas de las pantallas de los teléfonos móviles de los estudiantes que realizaban en prácticas en la Clínica Odontológica de una Universidad de Cuenca. La frecuencia de cada gen se muestra en la Tabla 2.
En las Figuras 1 a 5 se detallan las corridas electroforéticas de cada uno de los genes que codifican para hemolisinas.

\section{DISCUSIÓN}

Los teléfonos móviles son reservorios importantes de patógenos intrahospitalarios y comunitarios que producen infecciones causantes de problemas de salud. ${ }^{9,11}$

Este estudio detectó la presencia de los genes que codifican para hemolisinas de $S$. aureus; se detectaron hld y $h / g$ en $75 \%$, hla en $71 \%$, hlg-v en $69 \%$ y hlb en $25 \%$. Se interpretó que el gen más frecuente en las cepas aisladas de $S$. aureus en nuestro estudio fue $h / d$ y $h / g$.

En el estudio de Emira Noumi y colaboradores en 2020 en Túnez se aislaron 24 cepas de Staphylococcus spp. en teléfonos móviles de estudiantes; se detectó el gen hla en nueve cepas con $37.5 \%$, dato que es menor que en este estudio. ${ }^{12}$

Existen muy pocas investigaciones en las que se hayan detectado en teléfonos móviles todos los genes que codifican para hemolisinas, por lo que compararemos con cepas de $S$. aureus aisladas de otras procedencias.

En el estudio realizado por Rodríguez Acosta y colaboradores en 2010 en Paraguay se recolectaron muestras de secreciones, líquidos de punción y partes blandas. Se analizaron 50 genes aislados de $S$. aureus y se detectó el gen hla en $16 \%$ y hlb en $8 \%$, en comparación con nuestro estudio la detección de estos genes es menor. ${ }^{8}$

En el estudio de Kim, Ga Yeon y colaboradores en 2015 en Seúl y Cheonan se recolectaron muestras de saliva de pacientes con enfermedad periodontal, en las cuales estuvieron presentes los genes de virulencia que codifican para hemolisinas en las 41 cepas aisladas de $S$. aureus. Se detectó el gen hla en $97.6 \%$, hlb en $10 \%$, hld en $100 \%$, lo que coincide con nuestro estudio en relación a los genes que codifican para hemolisinas más frecuentes aisladas en cepas de $S$. aureus. ${ }^{13}$

\section{CONCLUSIÓN}

Los teléfonos móviles son una fuente de reservorio para S. aureus. Este dispositivo se usa para diferentes tipos de labores en el área de la salud, la falta de normas para su adecuado uso hace que estos equipos puedan diseminar cepas virulentas de $S$. aureus productoras de hemolisinas en las clínicas odontológicas.

Es importante desarrollar e implementar medidas específicas de información y prevención para evitar la contaminación de teléfonos móviles y de esta manera disminuir la diseminación de este importante patógeno. 
Gen: hla

Amplicón: 209 pb

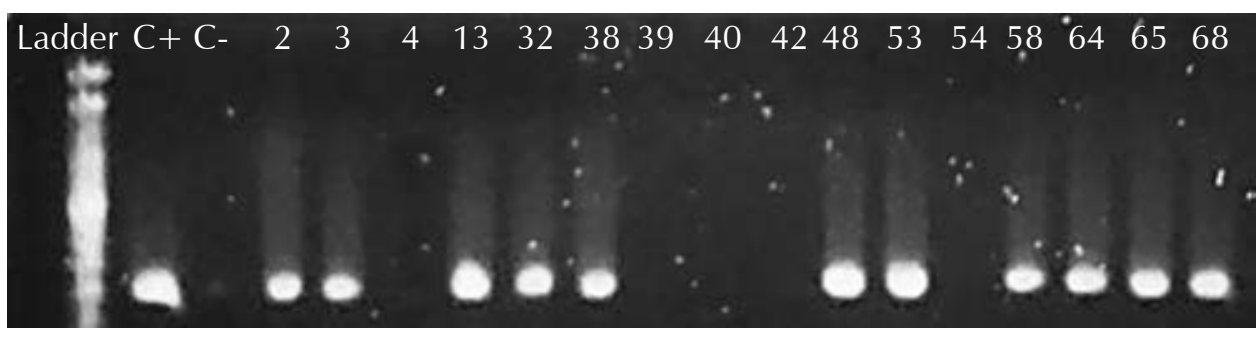

Figura 1: Producto de reacción en cadena de la polimerasa (PCR) para el gen hla (209pb) en S. aureus aislados en pantallas de celulares, carril 1 ladder, carril 2 control positivo cepa Staphylococcus aureus ATCC 25923, carril 3 control negativo cepa Streptococcus pyogenes ATCC 12344, muestras positivas 2, 3, 13, 32, 38, 48, 53, 58, 64, 65, 68, muestras negativas 4, 39, 40, 42, 54.

Gen: hlb

Amplicón: 309 pb

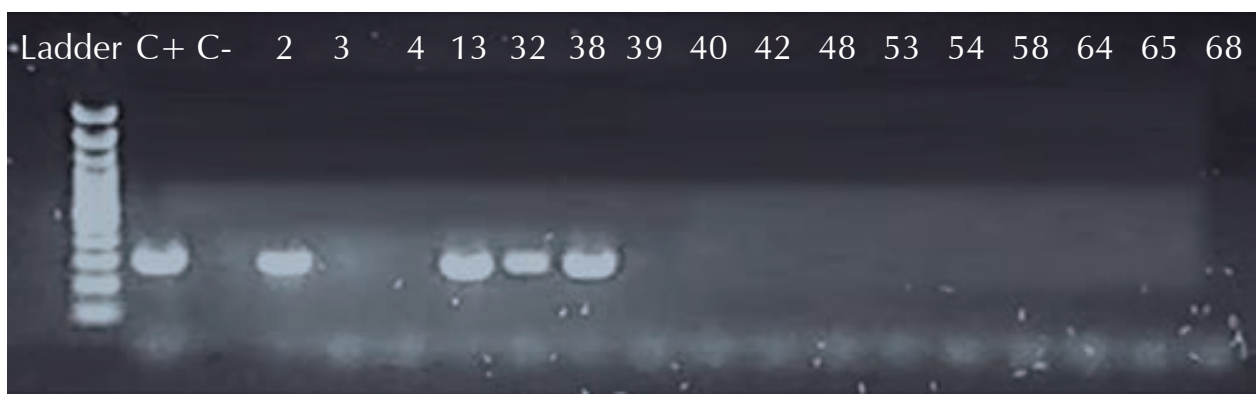

Figura 2: Producto de reacción en cadena de la polimerasa (PCR) para el gen hlb (309 pb) en S. aureus aislados en pantallas de celulares, carril 1 ladder, carril 2 control positivo cepa Staphylococcus aureus ATCC 25923, carril 3 control negativo cepa Streptococcus pyogenes ATCC 12344, muestras positivas 2, 13, 32, 38, muestras negativas 3, 4, 39, 40, 42, 48, 53, 54, 58, 64, 65, 68.

\author{
Gen: hld \\ Amplicón: 111 pb
}

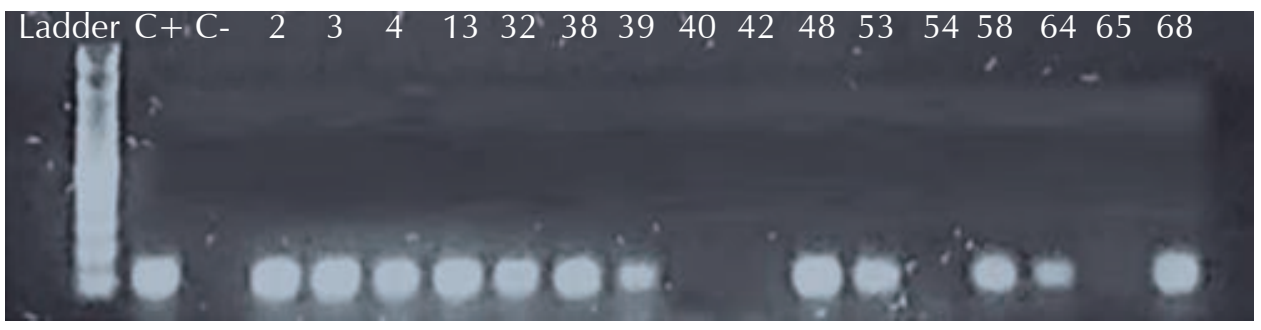

Figura 3: Producto de reacción en cadena de la polimerasa (PCR) para el gen hld (111 pb) en S. aureus aislados en pantallas de celulares, carril 1 ladder, carril 2 control positivo cepa Staphylococcus aureus ATCC 25923, carril 3 control negativo cepa Streptococcus pyogenes ATCC 12344, muestras positivas 2, 3, 4, 13, 32, 38, 39, 48, 53, 58, 64, 68, muestras negativas 40, 42, 54, 65. 


\section{Gen: $h l g$}

Amplicón: 535 pb

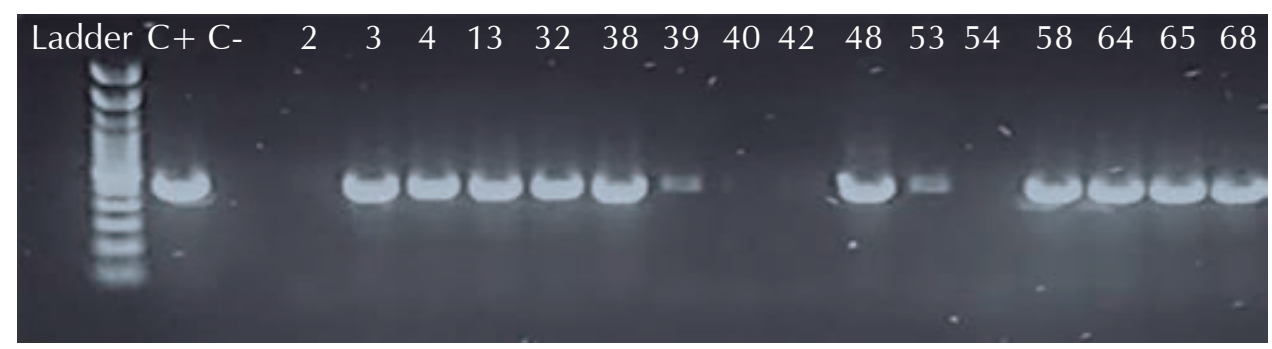

Figura 4: Producto de reacción en cadena de la polimerasa (PCR) para el gen hlg (535 pb) en S. aureus aislados en pantallas de celulares, carril 1 ladder, carril 2 control positivo cepa Staphylococcus aureus ATCC 25923, carril 3 control negativo cepa Streptococcus pyogenes ATCC 12344, muestras positivas $3,4,13,32,38,39,48,53,58,64,65,68$, muestras negativas 2, 40, $42,54$.

Gen: hlg-v

Amplicón: 390 pb

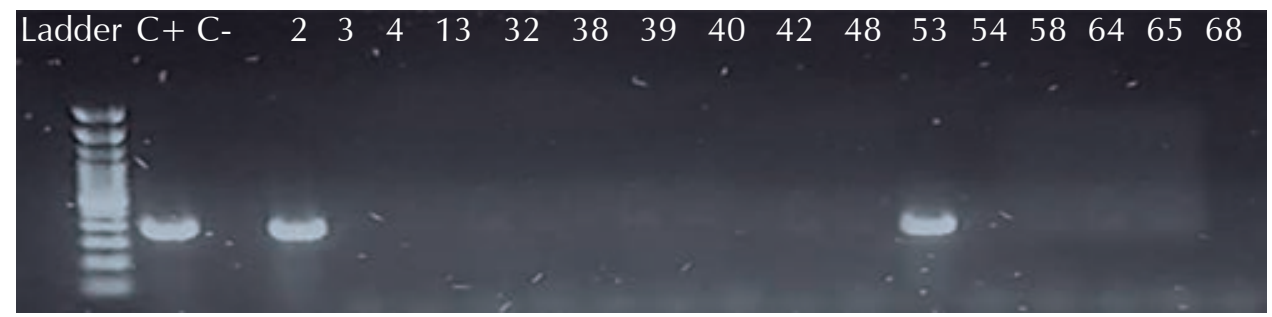

Figura 5: Producto de reacción en cadena de la polimerasa (PCR) para el gen hlg-v (390 pb) en S. aureus aislados en pantallas de celulares, carril 1 ladder, carril 2 control positivo cepa Staphylococcus aureus ATCC 25923, carril 3 control negativo cepa Streptococcus pyogenes ATCC 12344, muestras positivas 2, 53, muestras negativas 3, 4, 13, 32, 38, 39, 40, 42, 48, 54, 58, 64, 65, 68.

\section{AGRADECIMIENTOS}

Los autores expresan un agradecimiento especial a los directivos de la Universidad Católica de Cuenca por brindar acceso al Laboratorio de Genética y Biología Molecular, Basílica, CITT, donde se desarrolló la presente investigación.

\section{REFERENCIAS}

1. Lemus-Espinoza D, Lemus R, Maniscalchi BMT, Bónoli S. Contaminación bacteriana y fúngica en equipos de telefonía móvil en Barcelona, Estado Anzoátegui, Venezuela. Saber. 2015; 27 (4): 547-553.

2. Hernández-Orozco HG, Castañeda-Narváez JL, Garza EA. Celulares y riesgo de infecciones intrahospitalarias. Infectol Pediatr [Internet]. 2017; 30 (2): 45-47. Disponible en: http://www.medigraphic.com/ pdfs/infectologia/lip-2017/lip172a.pdf

3. Robinson FPA, Shalit M. The dezincification of brass. Anti-Corrosion Methods and Materials. 1964; 11 (4): 11-14.

4. Diana L, Ciuffo C, Musto H. Identificación y caracterización de Staphylococcus resistentes a meticilina aislados de perros. Veterinaria. 2019; 55 (212): 45-51.
5. Sonia RA, Maribel CG, Messaria GP, Armindo PM. Leucocidina de Panton Valentine en cepas SAMR aisladas de pacientes del Hospital Universitario de Maracaibo. Kasmera. 2016; 44 (2): 111-120.

6. Carrada LG, Castañón SCA. Quercetin attenuates Staphylococcus aureus virulence by reducing alpha-toxin secretion. Rev Argent Microbiol. 2018; 50 (2): 131-135.

7. Bustos-Martínez J, Hamdan-Partida A, Gutiérrez-Cárdenas M. Staphylococcus aureus: the reemergence of a pathogen in the community. Rev Biomed. 2006; 17 (4): 287-305.

8. Rodríguez AF, Carpinelli L, Basualdo W, Castro H, Quiñonez B, Argüello R et al. Frecuencia de genes que codifican factores de virulencia en Staphylococcus aureus aislados de niños que concurrieron al Hospital General Pediátrico Niños de Acosta Nú, durante el año 2010. Mem Inst Investig Cienc Salud. 2015; 13 (1): 58-66.

9. Andrade T C, Orellana B P. Frecuencia y susceptibilidad a penicilina y meticilina de aislamientos ambientales de Staphylococcus aureus en un hospital de Cuenca. Kasmera. 2019; 47 (2): 123-130.

10. Jarraud S, Mougel C, Thioulouse J, Lina G, Meugnier H, Forey F et al. Relationships between Staphylococcus aureus genetic background, virulence factors, agr groups (alleles), and human disease. Infect Immun. 2002; 70 (2): 631-641. 
11. Banawas S, Abdel-Hadi A, Alaidarous M, Alshehri B, Bin Dukhyil $\mathrm{AA}$, Alsaweed $\mathrm{M}$ et al. Multidrug-resistant bacteria associated with cell phones of healthcare professionals in selected hospitals in Saudi Arabia. Can J Infect Dis Med Microbiol. 2018; 2018: 6598918.

12. Noumi E, Merghni A, Alreshidi M, Campo R Del, Adnan M, Haddad $\mathrm{O}$ et al. Phenotypic and genotypic characterization with MALDITOF-MS based identification of Staphylococcus spp. Isolated from mobile phones with their antibiotic susceptibility, biofilm formation, and adhesion properties. Int J Environ Res Public Health. 2020; 17 (11): 3761.

13. Kim GY, Lee $\mathrm{CH}$. Antimicrobial susceptibility and pathogenic genes of Staphylococcus aureus isolated from the oral cavity of patients with periodontitis. J Periodontal Implant Sci. 2015; 45 (6): 223-228.
Conflicto de intereses: En la presente investigación los autores no presentan conflicto de interés.

Aspectos éticos: En la presente investigación no existen conflictos éticos, ya que la toma de muestras se realizó en objetos inanimados de los alumnos de último año de la carrera de odontología.

Financiamiento: Financiación mixta.

Correspondencia:

Nasly Elizabeth Hurtado Cantos

E-mail: nasly_hurtado_14@outlook.com 